\title{
Simplified analysis of organic compounds in headspace and aqueous samples by high- capacity sample enrichment probe
}

\author{
Ben V. Burger *, Brenda Marx, Maritha le Roux, Wina J.G. Burger \\ Department of Chemistry and Polymer Science, Stellenbosch University, Private Bag, Stellenbosch 7600, South Africa
}

\begin{abstract}
A sample enrichment probe (SEP) consisting of a thin rod of an inert material and provided at one end with a short sleeve of polydimethylsilicone rubber was used for the high-capacity sample enrichment of analytes from gaseous and aqueous samples for analysis by gas chromatography (GC) and its hyphenated techniques. The silicone rubber was exposed to the analytical sample, after which the end of the rod carrying the silicone rubber was introduced into the injector and the analytes thermally desorbed and analysed by GC. This technique is similar to, but differs from, solidphase microextraction (SPME) in that a much larger volume of the sorptive phase is employed, the sorptive phase is not introduced into the inlet of the GC via a needle and the injector is opened to the atmosphere for the introduction and removal of the SEP. In the determination of volatile and semi-volatile organic compounds in gaseous and aqueous media, the SEP technique gave results comparable with those obtained by the stir-bar-sorptive extraction (SBSE) and high-capacity sorption probe (HCSP) techniques. Implementation of the SEP technique requires only minor adaptations to the gas chromatograph and does not require any auxiliary thermal desorption and cryotrapping equipment.
\end{abstract}

Keywords: Headspace analysis; Environmental analysis; Water analysis; Sorptive sample enrichment; Silicone rubber; Sample enrichment probe (SEP).

\section{Introduction}

Despite the ability of gas chromatography $(\mathrm{GC})$ and its hyphenated techniques to detect picogram quantities of analyte, the selective enrichment of sample analytes has to be employed in order for these techniques to reach the limits of detection (LOD) and quantification (LOQ) required for biological and environmental analyses. The LOD can be reduced by large-volume injection [1], but only within limits. Solvent extraction of the analytes and concentration of the extract can also reduce LOD but it is time consuming, it is difficult to concentrate extracts without selectively losing the more volatile analytes, and solvents are expensive to dispose of. Using adsorbents or absorbents to selectively extract analytes from samples is an attractive alternative to solvent extraction, and various systems have been developed for this purpose [2] and [3]. One of the most popular is solid-phase microextraction (SPME) [4], which has gained wide acceptance because it is simple to use and its non-automated version does not require adaptation of the gas chromatograph or additional expensive instrumentation.

However, the enrichment that can be achieved with SPME is low because of the unfavourable partition between the microlitre volumes of sorbent on the fibre and large sample volumes. To increase the sensitivity of headspace analyses, Baltussen et al. [5] developed stir-bar-sorptive extraction (SBSE) [5] in which the analytes are enriched in a sorptive sleeve of rubber on a magnetic follower or stir-bar. This technique can be applied to both liquid and gas samples. Because a much larger volume of the sorptive stationary phase is used in this technique, the sensitivity of this method is much higher than that of SPME. Pettersson et al. [6] developed the high-capacity sorption probe (HCSP) in which a similarly large volume of the sorptive stationary phase is employed. These methods are flexible in as far as the volume of the sorptive medium is concerned, and they can therefore be adapted to a variety of applications.

Solid-phase aroma-concentrate extraction (SPACE) [7], in which volatiles are enriched in an absorbent consisting mainly of graphite-carbon coated on a stainless-steel rod, is another recently introduced sample enrichment method. Because a very small volume of sorptive phase is used in SPME, thermal desorption of the enriched material takes place almost instantaneously and the volatiles desorbed from the fibre need not be cryofocused on the column. The large volumes of sorptive material employed in SBSE, HCSP and SPACE preclude instantaneous desorption of the volatiles, which therefore have to be cryofocused on the column. This complicates implementation of these techniques and creates a niche for a simple method that can be used for analytical troubleshooting and for applications that do not require high-throughput, automated instrumentation. 
When possibilities of simplifying high-capacity sorptive sampling in trace gas chromatographic analysis are considered, it is important to realise that employing injector temperatures far below the boiling point of a high-boiling analyte does not necessarily have an adverse effect on the peak width with which the analyte is eluted from a column under otherwise normal conditions, provided the injector liner is clean and not packed with an adsorbent. Despite the slow vapourisation of such a compound at injector temperatures below its boiling point, it is quantitatively transferred to the column as long as the sample does not run down the liner to the bottom of the injector before it is completely vapourised. If in a temperature-programmed analysis, this process is completed before the elution temperature of the compound is reached, a high-boiling compound will elute with a peak shape similar to that obtained by injecting the sample at an injector temperature above its boiling point.

This principle is also applicable to the slow thermal desorption of heavy analytes from a large volume of sorptive phase. The critical problem in simplifying this type of analysis is thus not so much obtaining acceptable peak shapes for highboiling compounds but rather obtaining sharp peaks for volatile compounds that are not efficiently cold-trapped on the column. In this paper we report on the development of a simple sample enrichment probe (SEP) and a protocol for the analysis of volatiles from solid and aqueous samples without having to employ cryofocusing.

\section{Experimental}

\section{Instrumentation}

The standard-sized needle-guiding channels through the septum cap and septum-supporting insert of the split/splitless injector of a Carlo Erba HRGC 5300 gas chromatograph were enlarged using a 2.4-mm drill bit. The instrument was equipped with an FID. Data were acquired at a sampling rate of $25 \mathrm{~Hz}$ and were processed using Jasco-Borwin software, version 1.5. The following columns were used: glass open-tubular column A ( $40 \mathrm{~m} \times 0.3 \mathrm{~mm}$ I.D.), coated with $0.25 \mu \mathrm{m}$

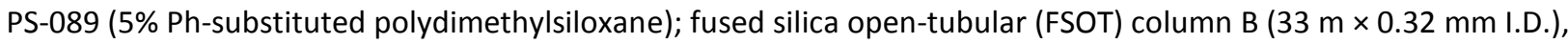
coated with $1.2 \mu \mathrm{m}$ PS-255 (100\% polydimethylsiloxane); glass open-tubular column C (40 m $\times 0.3 \mathrm{~mm}$ I.D.), coated with $2.5 \mu \mathrm{m}$ PS-255.

All analyses were carried out with hydrogen as carrier gas at a linear flow velocity of $50 \mathrm{~cm} / \mathrm{s}$, measured at an oven temperature of $40^{\circ} \mathrm{C}$. Low-resolution EI mass spectrometry (LR-EIMS) was performed on a Carlo Erba QMD $1000 \mathrm{GC}$ MS instrument using column A and helium as carrier gas at a linear velocity of $32.25 \mathrm{~cm} / \mathrm{s}$ at $40{ }^{\circ} \mathrm{C}$. The temperatures of the line-of-sight interface and ion-source were set at 250 and $180^{\circ} \mathrm{C}$, respectively. Mass spectra were recorded at $70 \mathrm{eV}$.

\section{SEP preparation}

SEPs were made according to the schematic drawing in Fig. $1 \mathrm{~A}$ using stainless-steel rods ( $130 \mathrm{~mm} \times 1.5 \mathrm{~mm}$ ), with one rounded end and a sharp point at the other. A section (15-mm length), about $3 \mathrm{~mm}$ from the rounded end, was machined to a diameter of $1.4 \mathrm{~mm}$. Laboratory-grade PDMS rubber tubing (1.00 mm I.D. × $1.75 \mathrm{~mm}$ O.D.) (Müller Labor Betrieb, Heidelberg) was cut into lengths of exactly $15.0 \mathrm{~mm}$ using a sharp blade. These sections of PDMS rubber tubing (PDMS sleeves) were weighed to five decimal places and only those sections with a mass of $0.02815 \pm 0.00005 \mathrm{~g}$ $( \pm 0.18 \%)$ were used as the sorptive elements of the SEPs.

Using ethanol as lubricating agent, the sleeves were slipped over the rounded tips of the rods and positioned on the narrower $(1.4-\mathrm{mm})$ sections of the rods. The resulting products were rolled between two glass plates to even out any differences in the thickness of the rubber that could have been caused by stretching or compressing the rubber during their installation on the rod. SEPs were also made by installing PDMS sleeves on glass rods and on lengths of fused silica (130 $\mathrm{mm} \times 0.7 \mathrm{~mm}$ O.D.), sealed off at both ends by means of an oxy-methane burner. These simple devices produced results similar to those obtained with SEPs made with stainless-steel rods.

\section{Ancillary hardware}

Performing SEP analyses does not require any hardware besides the sample vessels that are normally employed in sorptive sampling procedures. However, sampling is greatly facilitated by using sample vessels that can accommodate closures having the same thread as the septum cap of the gas chromatograph. The following simple hardware was therefore made. Commercially available 25-, 50-, and 100-ml glass bottles with interchangeable phenolic caps (Barloworld Scientific, Stone, UK) were chosen as standard sample bottles. The Teflon-faced rubber septum of one of these bottle caps was removed and a hole $(13 \mathrm{~mm})$ was drilled through the centre of the cap. A tubular stainless-steel insert (15 mm $\times 13 \mathrm{~mm}$ O.D. $\times 2.4 \mathrm{~mm} \mathrm{I.D.)}$ (Fig. 1B), having the same 13-mm thread as the injector of the GC, was made. Care was taken to ensure the tightest possible fit of the metal insert into the hole in the phenolic cap. The insert was pressed into the hole and some epoxy glue was applied to the outside surface of the cap, around the insert, to ensure a gas-tight seal. 
The glue was allowed to harden, after which the cap was heated overnight in a $\mathrm{GC}$ oven at $80{ }^{\circ} \mathrm{C}$ to remove any residual volatiles. This bottle cap was fitted with a gasket made from 0.4- $\mathrm{mm}$ Teflon. Fig. $1 \mathrm{~B}$ is a schematic drawing of the finished product as it was used for the sampling of, for example, the headspace volatiles of a beverage. It was convenient to have available several of these bottle and septum caps. For larger gas samples of up to $10 \mathrm{I}$, the neck of one of the sample bottles with its screw thread was removed from the bottle and was fused to a B-29 ground-glass cone. This connecting piece made it possible to use the phenolic bottle cap with its insert on larger flasks with standard ground-glass joints. If, on the other hand, small vials were required, their relatively cheap septa were pierced by using the sharp end of the SEP. These septa were discarded after use.

\section{Chemicals and standard solutions}

A standard gas sample containing $14 n$-alkanes from $C_{5}$ to $C_{18}$ in concentrations of approximately $1 \mu \mathrm{g} / \mathrm{l}$ was prepared by diluting $1.0 \mu \mathrm{l}$ of a mixture of the pure hydrocarbons in $10 \mathrm{I}$ of high purity argon, which had been further purified by passing it over $\mathrm{CuO}$ at $700{ }^{\circ} \mathrm{C}$. A solution containing the 30 chemical pollutants listed in Table 3 (Sigma-Aldrich, Cape Town, South Africa) in concentrations of approximately $1 \mu \mathrm{g} / \mathrm{ml}$ in dichloromethane/benzene (3:1) was diluted in methanol to give a stock solution containing each of the compounds in a concentration of $1 \mathrm{ng} / \mathrm{ml}$. Appropriate dilution of this solution with deionised water gave a solution containing each of the analytes in a concentration of approximately $1 \mathrm{ng} / \mathrm{l}$.

The resulting solution was spiked with $10 \mu \mathrm{g}$ hyamine [8] (Fluka product from Sigma-Aldrich, Cape Town), and $100 \mathrm{~g}$ quantities of the resulting solution were weighed out to four decimal places for relative standard deviation (RSD) determinations by SEP-GC/FID.

\section{SEP installation and conditioning}

A standard Carlo Erba glass injector liner (open type, I.D. $4 \mathrm{~mm}$ ) was installed in the injector of the GC. The bevelled tip of a 1.2-mm stainless-steel needle was removed; the remaining part of the needle was sharpened and then used to punch a small hole in the middle of a Teflon-faced septum. This was done to avoid tearing the Teflon during the next operation. The pointed end of the shaft was inserted into the hole from the Teflon side and the septum was moved on the shaft to position the PDMS sleeve about halfway into the injector liner when the SEP was installed in the injector. This position was measured and noted for future use. Any type of Teflon-faced septum can be used, but septa faced with relatively thick Teflon (SGE, Ringwood, Australia, cat. no. 041882) are preferred because the thick Teflon is folded back into the rubber when the shaft of the SEP is inserted into the septum to create a practically gas-tight seal between the Teflon and the shaft, preventing exposure of the silicone rubber to the analytes.

The SEP was installed in the injector of the GC using the septum cap with its enlarged central hole (Fig. 2) and conditioned with the carrier gas turned on for an hour or longer at a temperature not higher than $230{ }^{\circ} \mathrm{C}$. After appropriate conditioning, the SEP was removed from the injector. Without removing the septum or the septum cap from the stainless-steel shaft, the rounded end of the SEP was inserted into a narrow glass tube up to the position of the septum, to restrict contact of the adsorbent sleeve with laboratory air. After it had cooled down to room temperature the SEP was ready for sample enrichment. If preferred, the conditioned SEP may be sealed off in a glass tube or stored in a glass tube with a standard B5 ground-glass closure.

\section{Standard sampling and analytical procedure}

A liquid sample for headspace analysis was placed in a standard sample bottle with an adapted phenolic cap. The preconditioned SEP with its septum and septum cap already in place was introduced into the headspace of the sample via the metal insert of the phenolic cap, the septum cap was tightened to ensure gas-tight closure of the sample bottle and the sample was stirred at an appropriate temperature using a glass-encapsulated stir bar (Fig. 1B). After completion of sampling, the SEP was removed from the sample vessel. When necessary, the position of the septum on the shaft of the SEP was readjusted to the position it had in the conditioning step.

With the carrier gas of the GC turned off, the standard septum cap and septum were removed from the injector and the SEP was installed in the injector (Fig. 2) by allowing it to drop into the injector, which ensured that the PDMS sleeve passed through the hot septum-supporting insert of the injector as rapidly as possible. This was done to avoid losing highly volatile analytes. The septum cap was tightened, the carrier gas was turned on after a delay of about $15 \mathrm{~s}$, and the temperature programme and data acquisition were started. At the end of the analysis the GC oven was allowed to cool down and the SEP was removed and stored in a glass tube. If the previous sample did not contain any high-boiling compounds the SEP was used for the next analysis without further conditioning.

Gas samples and the headspace gas of solids were sampled and analysed in a similar manner. 


\section{Results and discussion}

The Section 1 mentions that the peak shape with which a high-boiling compound is eluted from a column is not adversely affected when the injector temperature is much lower than the boiling point of the analyte. This was experimentally verified in a GC analysis of a solution of octadecane in dichloromethane using an injector temperature of $150{ }^{\circ} \mathrm{C}$, a split flow of $15 \mathrm{ml} / \mathrm{min}$ and a programming rate of $4{ }^{\circ} \mathrm{C} / \mathrm{min}$. Octadecane, which has a boiling point of $317^{\circ} \mathrm{C}$, about $170^{\circ} \mathrm{C}$ higher than the injector temperature, was eluted with perfect peak shape (peak asymmetry of 0.98). Tailing of the octadecane peak only became noticeable at injector temperatures lower than $100{ }^{\circ} \mathrm{C}$. It was therefore argued that although the desorption of volatiles from larger volumes of PDMS rubber is much slower than desorption from an SPME fibre, instantaneous desorption of the high-boiling analytes from a large volume of rubber is not a prerequisite for perfect gas chromatography of such analytes.

High-boiling compounds are immobilised in the stationary phase of the column during the earlier stages of the temperature programme when the column is still far below the elution temperature of these compounds, provided that the temperature programming rate is low enough to allow for the complete desorption of the analytes well before the elution temperatures of the analytes in question are reached. The critically important problem in high-capacity sorptive sampling is that highly volatile analytes are not efficiently trapped and retained on the column during the time required for complete desorption of the high-boiling analytes from the sorptive medium. In other systems, this problem is eliminated by cryotrapping.

However, it was thought that it might be possible to circumvent the cryotrapping of volatiles if instantaneous thermal desorption of highly volatile analytes could be achieved. This possibility was investigated using a sample enrichment probe with integrated resistive heating of the PDMS. Although the section of the probe carrying the PDMS sleeve could be heated to high temperatures within fractions of a second using this device, alkanes from pentane to octane were eluted as two partly merged peaks. The higher-boiling alkanes were eluted with acceptable peak shapes. This was an unexpected and totally unacceptable result, and this approach was abandoned. However, the results gave rise to the question as to whether it might be possible to find conditions that would increase the size of the first peak at the cost of the second so that these volatile compounds would be eluted as single peaks with acceptable peak shapes.

The results of experiments carried out towards this end, using column A, are shown in Fig. 3A-E. In the first experiment the injector was operated in the splitless mode at a temperature of $220^{\circ} \mathrm{C}$. In the resulting chromatogram (Fig. $\left.3 \mathrm{~A}\right)$, pentane, hexane, heptane and nonane each eluted as very broad, partly resolved, double peaks, showing strong tailing, while decane was eluted with only a barely visible shoulder at its apex. The splitting of the peaks of the lower alkanes did not result from poor column surface deactivation and/or stationary phase film quality, but was due to the desorption of the analytes from the PDMS rubber in two stages. This phenomenon can be rationalised by taking into account that the carrier gas has to be turned off when the SEP is introduced into the injector to avoid premature desorption and loss of the volatiles.

The first sharp peak of each pair of peaks in Fig. 3A therefore represents the material desorbed from the rubber during these few seconds after introduction of the SEP into the injector, before the carrier gas was turned on again. This material was transported to the column as a sharp band. The second, broader member of each pair of peaks resulted from desorption of the remainder of that particular compound. Due to the inefficient cold trapping on the column the two bands of each of the volatile analytes arriving in the column migrated as they arrived in the column and were eluted as two partly resolved peaks, with different peak shapes. The chromatogram in Fig. 3B was obtained at an injector temperature of $300^{\circ} \mathrm{C}$. Although the decane was eluted with a decreased peak width in this chromatogram, it was clear that increasing the injector temperature would not solve the problem.

As expected, the tailing of the peaks decreased by operating the injector in the split mode (Fig. 3C). The size of the second peak of each of the peak pairs was decreased, which resulted in additional merging of the peak pairs. Because nonane and decane are cold trapped more efficiently than the lighter alkanes they are eluted in this analysis with reasonable peak symmetries of 2.21 and 1.77 , respectively. In these two analyses a split flow of $15 \mathrm{ml} / \mathrm{min}$ was used. The splitting of peaks was practically eliminated by using a split flow of $30 \mathrm{ml} / \mathrm{min}$. However, all of the $\mathrm{C}_{5}-\mathrm{C}_{10}$ peaks still showed unacceptable tailing (Fig. 3D).

It was argued that the size of the first peak of each pair should increase at the cost of the size of the second if a larger proportion of each of the volatiles is allowed to desorb from the rubber before the carrier gas is turned on, which could result in a complete merging of the peak pairs. Extending the flowless period (period during which the carrier gas was turned off) to $15 \mathrm{~s}$ produced far better results; the $\mathrm{C}_{5}-\mathrm{C}_{8}$ peaks had only small shoulders on their tailing edges (Fig. $3 \mathrm{E}$ ). Extending the flowless period to $30 \mathrm{~s}$ gave no appreciable improvement. As anticipated, using columns with thicker stationary phase films solved the problem of the remaining tailing of the early-eluting peaks. Column B with a film thickness of $1.2 \mu \mathrm{m}$ gave peak asymmetries of 1.39, 1.45, 1.28 and 1.06, respectively, for the early-eluting alkanes from 
pentane to octane. A flowless period of $30 \mathrm{~s}$ instead of $15 \mathrm{~s}$ produced only marginally better asymmetries of $1.30,1.20$, 1.20 and 1.09, respectively. It therefore did not seem worthwhile to increase the flowless period beyond about $15 \mathrm{~s}$. SEP enrichment of $n$-alkanes from the standard gas sample mentioned above and GC analysis on the apolar column B gave the gas chromatogram depicted in Fig. 4A.

A series of these analyses gave acceptably low relative standard deviations for all of the alkanes except pentane and hexane. Using column $\mathrm{C}$ with its higher film thickness of $2.5 \mu \mathrm{m}$ and a retention gap gave slightly lower RSDs for pentane and hexane, but a much higher RSD for octadecane. The relevant data are given in Table 1. The same SEP was used for all of the analyses in each of the two experiments. The bell-shaped elution profile in Fig. 4A can be ascribed to a combination of non-equilibrium enrichment (to avoid overloading the system), some loss of the more volatile alkanes when the gas sample was made up, and the lower molar responses of the short-chain alkanes. The products of the thermal degradation of PDMS rubber are known compounds that elute as sharp peaks, and do not present a problem in GC techniques in which thermal desorption from PDMS rubber is employed.

In Fig. 4B a typical gas chromatogram obtained in a blank analysis with a freshly conditioned SEP illustrates that even laboratory-grade PDMS rubber undergoes very little degradation at the low injector temperatures that are used in this technique. In trace analysis this is an additional advantage of the SEP technique. Because sample enrichment is the most time-consuming step in trace headspace analysis, regardless of the sample enrichment technique that is used, the possibility of saving time by the simultaneous sample enrichment from the same sample with multiple SEPS was investigated. Ten SEPs were simultaneously introduced into and removed from the standard $n$-alkane gas sample. On removal from the sample, the SEPs were immediately inserted into thin glass tubes and sealed off.

Each SEP was removed from its glass tube immediately before it was introduced into the injector. The results were very disappointing; RSDs varied from about $12 \%$ for pentane to $6 \%$ for decane and about $22 \%$ for the higher alkanes, hence the experiment was aborted after six runs. These results cannot be attributed to the loss of the lighter as well as the heavier hydrocarbons during transfer of the SEPs to and from the glass tubes or to poor reproducibility of the thermal desorption of the analytes. Over a period of several months some of the SEPs used in this experiment had been subjected to conditioning at temperatures between 220 and $300^{\circ} \mathrm{C}$ for periods varying from a few hours to several days.

An explanation for the poor results obtained in this experiment, and in several further experiments, could therefore be that PDMS rubber undergoes changes when it is subjected to harsh thermal treatment and that these changes influence the capacity of the rubber and/or the rate at which compounds are sorbed into the rubber under nonequilibrium sampling conditions. As mentioned above, the results reported in Table 1 were obtained using the same SEP for all the analyses in each of the two experiments. Although better results were obtained when SEPs with similar thermal histories were used in the simultaneous sampling experiments, using the same SEP in replicate analyses always gave better results. If SEPS are numbered, a complete thermal record is kept of each of them, and SEPs with similar thermal histories are used in such simultaneous sampling experiments, this problem could perhaps be kept within reasonable limits.

This phenomenon has not yet been investigated in sufficient detail to suggest other possible explanations and solutions. Although the SEP technique offers a simple alternative to other trace methods of analysis there are two valid questions that have to be answered, specifically with regard to ways in which this technique differs from SBSE and HCSP. The first question is whether, in a temperature-programmed analysis, the desorption of a high-boiling compound such as octadecane will be completed in good time for the production of sharp peaks before its elution temperature is reached. Using the standard $n$-alkane gas sample, this aspect was investigated by removing the SEP from the injector immediately after octadecane had been eluted, without cooling down the column.

This SEP was then immediately subjected to another desorption and analysis cycle. With the exception of relatively small quantities of hexadecane, heptadecane and octadecane, all of the alkanes were quantitatively desorbed in the first cycle. The percentages of these three compounds carried over to the second cycle at temperature-programming rates of 4 and $32{ }^{\circ} \mathrm{C} / \mathrm{min}$ are given in Table 2 . At a ramp rate of $4{ }^{\circ} \mathrm{C} / \mathrm{min}$ from 30 to $270{ }^{\circ} \mathrm{C}$, octadecane was eluted at a retention time of $56.7 \mathrm{~min}$ and the SEP was removed from the injector at a run-time of $60 \mathrm{~min}$, whereas at a ramp rate of $32^{\circ} \mathrm{C} / \mathrm{min}$ from 30 to $270^{\circ} \mathrm{C}$ octadecane was eluted at $16.3 \mathrm{~min}$ during the isothermal stage of the temperature programme, and the SEP was removed at a run-time of $20 \mathrm{~min}$. The SEP thus spent a much shorter time at $230{ }^{\circ} \mathrm{C}$ in the latter analysis and therefore larger quantities of the higher alkanes were found in the second analytical cycle.

It is surprising that such widely differing programming rates did not result in even larger differences in the undesorbed high-boiling analytes found in the second cycle. Nevertheless, it is clearly not advisable to use very high programming rates in SEP analyses. Because standard operating procedures require the SEP to be left in the injector while the oven cools down, any reasonably volatile material still present in the PDMS rubber at the end of the temperature 
programme or after the final isothermal period will be desorbed while the oven is cooling down. It is thus not necessary to subject the SEP to additional conditioning, provided the sample does not contain heavy material that could build up in the rubber. When, for example, the oven was cooled down from 280 to $40{ }^{\circ} \mathrm{C}$ at $10{ }^{\circ} \mathrm{C} / \mathrm{min}$ the $\mathrm{C}_{16}-\mathrm{C}_{18}$ alkanes were not detected in the second analysis. However, had any residual material been desorbed during the cooling period, the material would have been trapped on the column, thus requiring additional conditioning of the column.

Consequently, the best solution is to allow sufficient time at the final column temperature for complete desorption and elution of the residual analytes or other constituents that could negatively affect subsequent analyses. Depending on the application, other measures can be taken to solve this problem. For example, a somewhat higher injector temperature or a lower temperature-programming rate could be used. A second matter of concern is the introduction of air into the injector in addition to the air that is taken up in the PDMS rubber during sampling. The question is: how much air is introduced into the system and how does the hot air, albeit diluted with the carrier gas, impact on sensitive compounds at temperatures higher than $200^{\circ} \mathrm{C}$.

In GC-MS experiments in which the SEP was introduced into the injector as rapidly as possible, the air peak in the total ion chromatogram (TIC) had a width (wb) of $48 \mathrm{~s}$. In SPME analyses only the relatively small volume of air present in the needle of the SPME device is released into the injector. This air is rapidly flushed from the injector if split injection is employed. However, for some inexplicable reason, air diffuses into the injector as long as the needle of this device is left in the injector. We have observed this in GC-MS analyses with various PDMS fibres since the introduction of the SPME technique. It was demonstrated by a blank SPME analysis using a 100- $\mu \mathrm{m}$ PDMS fibre. In this experiment data acquisition was started immediately after introduction of the fibre into the injector and the fibre was arbitrarily removed after $2.5 \mathrm{~min}$.

The dead time of the column was $2.0 \mathrm{~min}$ and the elution of the air present in the SPME needle was observed starting from $2 \mathrm{~min}$. The effect of the removal of the SPME needle at $2.5 \mathrm{~min}$, indicated by a rapid decrease in the air signal, was observed at $4.5 \mathrm{~min}$. If, in such an analysis the needle is not removed, the elution of air continues throughout the analysis at about the same level. By integration of the air peak, the total quantity of air introduced into the injector during this 2.5-min period in the SPME analysis was determined to be only about $8 \%$ of that present in the injector during the $48 \mathrm{~s}$ period (width of air peak) in the SEP analysis. In order to compare the effect of the presence of air in the injector on sensitive compounds in SPME and SEP analyses, purified argon spiked with $\alpha$-pinene, $\gamma$-terpinene and limonene was analysed by these two techniques at injector temperatures of 180,220 and $240{ }^{\circ} \mathrm{C}$.

Even at an injector temperature of $240^{\circ} \mathrm{C}$ the two techniques gave identical results, with no indication of thermal or oxidative conversion of any of the analytes. It could be argued that because these terpenoids are not desorbed very rapidly, they could be less prone to oxidative conversion than the more volatile compounds that are desorbed during the first few seconds after introduction of the SEP into the injector while the concentration of air in the injector is at its highest. The oxidative conversion of the more volatile 1-butanethiol to dibutyl disulphide was therefore chosen as a probe for the determination of the effect of hot air on sensitive compounds. Split injection of $4 \mu$ of a gas sample containing $1 \mathrm{mg}$ of pure 1-butanethiol in $10 \mathrm{I}$ of purified argon gave a 1-butanethiol to dibutyl disulphide ratio of 99.5:0.5\%.

In SPME analyses of this gas sample (enrichment, $10 \mathrm{~min}$ at $22^{\circ} \mathrm{C}$ ) in which the fibre was left in the injector for 2,5 and $15 \mathrm{~min}$, respectively, thiol:disulphide ratios varying between 67.3:32.7 and 69.7:30.3 were obtained. SEP analyses of the gas sample (enrichment, $1 \mathrm{~min}$ at $22^{\circ} \mathrm{C}$ ) gave a ratio of $92.7: 7.3 \%$. In all of these analyses an injector temperature of $230^{\circ} \mathrm{C}$ was used. The results of these experiments show that the presence of oxygen in the injector during the early stages of an analysis has considerably less effect in SEP than in SPME analyses, at least on this thiol. These experiments were done in the above order, i.e. the SEP analysis was done last, so that the higher percentage of the disulphide found in the SPME analyses cannot be attributed to ageing of the gas sample.

The selection of the desorption conditions in SEP analysis is largely determined by the boiling point range of the analytes. Analytes with boiling points higher than about $170^{\circ} \mathrm{C}$ do not require the implementation of a flowless period, and such compounds are therefore affected even less by the introduction of air into the injector than low boiling compounds such as this thiol. In Fig. 5A and B the TICs are compared of SEP and SPME analyses of the very faint aroma of dry plant material of the herbal tea Aspalathus linearis, also known as Rooibos tea. SEP-GC-MS analysis of the headspace volatiles of the plant material enriched for $24 \mathrm{~h}$ at $40^{\circ} \mathrm{C}$ and GC-MS analysis using column A, gave the TIC shown in Fig. 5A and made available library-searchable or interpretable mass spectra of 140 of the constituents of the aroma of this tea.

SPME-GC-MS analysis employing the same sampling conditions, gave the TIC shown in Fig. 5B, which contains only a few peaks of diagnostic value. The SEP technique was developed primarily for headspace analysis. However, it can also be used for the enrichment of analytes from aqueous matrices, as illustrated by the GC-SEP/FID analysis of $100 \mathrm{~g}$ water 
samples spiked with 30 pollutants at concentrations of approximately $1 \mathrm{ng} / \mathrm{l}$ (Fig. 6). Although no attempt was made to optimise the conditions for the enrichment of the analytes, RSDs ranging from 2.0 to 9.8 were obtained (Table 3), comparable with the results Petterson et al. [6] obtained by using their HCSP technique. Bis(2-ethylhexyl)phthalate and benzo[ghi]perylene were outliers with RSDs of 20.7 and $50.0 \%$, respectively.

\section{Concluding remarks}

In SEP analyses of analytes with boiling points higher than about $170^{\circ} \mathrm{C}$, the thickness of the stationary phase film of the column is of minor importance. If, on the other hand, the sample contains more volatile analytes, the highest film thickness that will not unduly prolong the analysis time should be used. Normally it is not necessary to use injector temperatures much higher than $230^{\circ} \mathrm{C}$ for the desorption of analytes that are accessible in headspace analysis. At this temperature only negligible thermal degradation of the PDMS rubber was observed. However, the analysis of highboiling compounds such as polar water pollutants, for example, may require higher injector temperatures.

In principle, the SEP, SPME, SBSE and HCSP techniques differ only in that desorption and separation run more or less concurrently in SEP and SPME analyses, whereas in the latter two techniques the analytes are quantitatively desorbed from the sorptive medium before the gas chromatographic separation of the volatiles commences. Consequently, the SEP technique does not require cryotrapping of the volatiles desorbed from the sorptive medium. This is one major advantage of the SEP technique. An additional advantage of importance in some instances is that in SEP analyses the carrier gas flow cannot be interrupted by ice formation in the column, a problem that could be encountered if the small quantities of moisture taken up in the PDMS rubber during sampling are cryotrapped on the column.

Implementation of the SEP technique on certain gas chromatographs requires only minor inexpensive changes to the injector hardware. Other currently available gas chromatographs could, however, have more sophisticated injectors, which may require the replacement of the septum cap and the septum-supporting hardware with hardware that may have to be manufactured in an instrument maker's workshop. An automated version of the SEP technique has not been discussed in this paper, specifically to avoid the impression that the SEP technique requires certain skills or workshop facilities, an impression that could deter analysts from considering implementation of the technique when, on occasion, an application calls for high-capacity sample enrichment.

\section{Acknowledgments}

The research was supported by Stellenbosch University and the National Research Foundation, Pretoria, South Africa. 


\section{Tables}

Table 1

Area count repeatability for SEP-GC analyses of gas samples containing $n$-alkanes at concentrations of ca. $1 \mu \mathrm{g} / \mathrm{I}^{\mathrm{a}}$

\begin{tabular}{|c|c|c|}
\hline Alkanes & Experiment $\mathbf{1}^{\mathrm{b}}$ & Experiment $2^{\mathrm{c}}$ \\
\hline & RSD $(\%, n=5)$ & $\operatorname{RSD}(\%, n=5)$ \\
\hline$C_{5}$ & 5.99 & 2.05 \\
\hline $\mathrm{C}_{6}$ & 7.49 & 2.15 \\
\hline C7 & 3.76 & 1.96 \\
\hline $\mathrm{C}_{8}$ & 3.24 & 3.59 \\
\hline$C_{9}$ & 2.44 & 3.42 \\
\hline $\mathrm{C}_{10}$ & 2.44 & 4.32 \\
\hline $\mathrm{C}_{11}$ & 3.00 & 4.53 \\
\hline$C_{12}$ & 3.81 & 4.44 \\
\hline$C_{13}$ & 4.66 & 3.92 \\
\hline $\mathrm{C}_{14}$ & 4.08 & 2.85 \\
\hline $\mathrm{C}_{15}$ & 1.46 & 1.62 \\
\hline $\mathrm{C}_{16}$ & 3.42 & 0.49 \\
\hline $\mathrm{C}_{17}$ & 5.86 & 3.89 \\
\hline $\mathrm{C}_{18}$ & 5.60 & 9.60 \\
\hline
\end{tabular}

a The same SEP was used in each of the two experiments; sample enrichment in both experiments 10 min at $22{ }^{\circ} \mathrm{C}$. b FSOT column B, $33 \mathrm{~m} \times 0.32 \mathrm{~mm}$ I.D. $\times 1.2 \mu \mathrm{m}$ PS-255, programmed at $4{ }^{\circ} \mathrm{C} / \mathrm{min}$ from 40 to $250^{\circ} \mathrm{C}$ (hold $30 \mathrm{~min}$ ); injector $230^{\circ} \mathrm{C}$; flowless $15 \mathrm{~s}$; split $15 \mathrm{ml} / \mathrm{min}$.

c Glass open-tubular column C, $40 \mathrm{~m} \times 0.3 \mathrm{~mm}$ I.D. $\times 2.5 \mu \mathrm{m}$ PS-255, programmed at $4{ }^{\circ} \mathrm{C} / \mathrm{min}$ from 30 to $270{ }^{\circ} \mathrm{C}$; sample enrichment $10 \mathrm{~min}$ at $22{ }^{\circ} \mathrm{C}$; injector $250^{\circ} \mathrm{C}$ for $20 \mathrm{~min}$ and then $220^{\circ} \mathrm{C}$ until the end of the analysis; flowless $15 \mathrm{~s}$; split $15 \mathrm{ml} / \mathrm{min}$. 
Table 2

Material left undesorbed in a SEP at different injector temperatures and column temperature-programming rates

\begin{tabular}{|c|c|c|}
\hline \multirow{2}{*}{ Alkanes } & \multicolumn{2}{|c|}{ Average carry-over ${ }^{a, b}(n=3)$} \\
\hline & $\begin{array}{l}\text { Temperature gradient } \\
\left(4^{\circ} \mathrm{C} / \mathrm{min}\right)^{\mathrm{c}}\end{array}$ & $\begin{array}{c}\text { Temperature gradient } \\
\left(32^{\circ} \mathrm{C} / \mathrm{min}\right)^{\mathrm{d}}\end{array}$ \\
\hline$C_{5}-C_{15}$ & 0.00 & 0.00 \\
\hline$C_{16}$ & 0.39 & 0.64 \\
\hline$C_{17}$ & 0.93 & 1.11 \\
\hline $\mathrm{C}_{18}$ & 1.24 & 2.04 \\
\hline
\end{tabular}

a As \% of quantities desorbed in the first cycle.

b Glass open-tubular column A, $40 \mathrm{~m} \times 0.3 \mathrm{~mm}$ I.D. $\times 0.25 \mu \mathrm{m}$ PS-089; injector $220^{\circ} \mathrm{C}$; flowless $15 \mathrm{~s}$; split $15 \mathrm{ml} / \mathrm{min}$. c Temperature gradient $4{ }^{\circ} \mathrm{C} / \mathrm{min}$ from 30 to $270{ }^{\circ} \mathrm{C}$.

d Temperature gradient $32^{\circ} \mathrm{C} / \mathrm{min}$ from 30 to $270^{\circ} \mathrm{C}$.

Table 3.

SEP analysis of water spiked with 30 pollutants at concentrations of approximately $1 \mathrm{ng} / \mathrm{l}$

\begin{tabular}{|c|c|c|}
\hline No. in Fig. 6 & Compound & RSD (\%) \\
\hline 1 & 1,3-Dichlorobenzene & 9.74 \\
\hline 2 & 1,4-Dichlorobenzene & 4.74 \\
\hline 3 & 1,2-Dichlorobenzene & 5.57 \\
\hline 4 & 1-Bromo-2-methylbenzene & 7.98 \\
\hline 5 & Bis(2-chloroisopropyl)ether & 9.62 \\
\hline 6 & 1,2,4-Trichlorobenzene & 3.43 \\
\hline 7 & Naphthalene & $\mid 7.12$ \\
\hline 8 & 2-Chloronaphthalene & 4.64 \\
\hline 9 & Acenaphthylene & 4.29 \\
\hline 10 & Acenaphthene & 4.14 \\
\hline 11 & 2,4-Dinitrotoluene & $\mid 5.20$ \\
\hline 12 & Fluorene & $\mid 7.64$ \\
\hline 13 & 4-Chlorophenyl phenyl ether & 4.57 \\
\hline 14 & Azobenzene & 7.42 \\
\hline 15 & 4-Bromophenyl phenyl ether & 4.81 \\
\hline 16 & Hexachlorobenzene & 1.97 \\
\hline
\end{tabular}




\begin{tabular}{|c|c|c|}
\hline No. in Fig. 6 & Compound & RSD (\%) \\
\hline 17 & Phenanthrene & 6.88 \\
\hline 18 & Anthracene & 6.82 \\
\hline 19 & Carbazole & 8.86 \\
\hline 20 & Di-n-butylphthalate & 9.60 \\
\hline 21 & Fluoranthene & 7.85 \\
\hline 22 & Pyrene & 8.08 \\
\hline 23 & Butyl benzyl phthalate & 6.20 \\
\hline 24 & Benzo[a]anthracene & 9.45 \\
\hline 25 & Chrysene & 8.87 \\
\hline 26 & Bis(2-ethylhexyl)phthalate & 20.68 \\
\hline 27 & Benzo[b]fluoranthene & $\mid 5.87$ \\
\hline 28 & Benzo[k]fluoranthene & 6.16 \\
\hline 29 & Benzo[a]pyrene & 9.82 \\
\hline 30 & Benzo[ghi]perylene & 51.95 \\
\hline
\end{tabular}




\section{Figures}

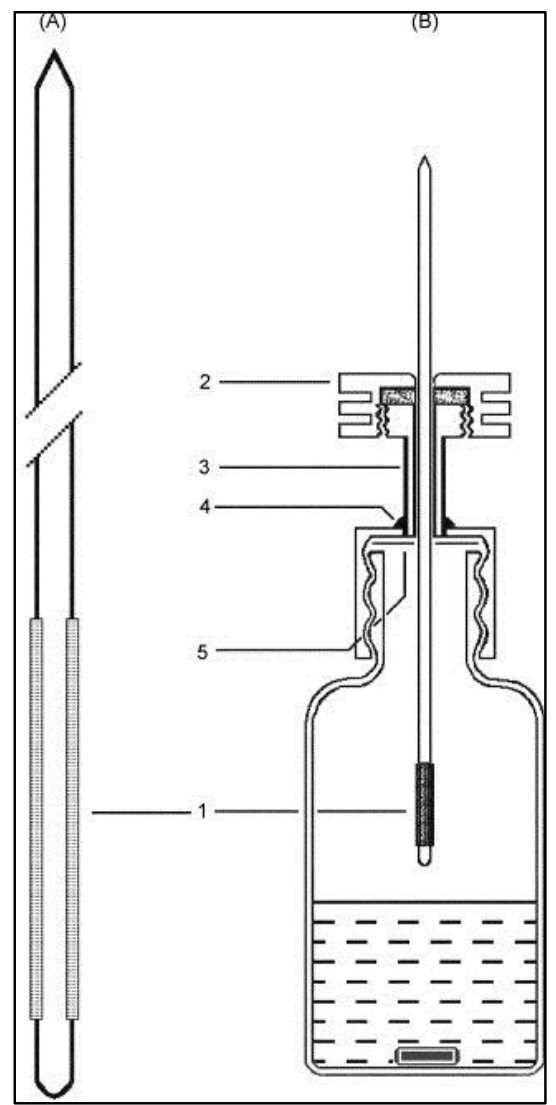

Fig. 1. (A) Sample enrichment probe (SEP) made from stainless steel with (1) a 15-mm sleeve of PDMS rubber; (B) sample bottle with a SEP installed for enrichment of headspace volatiles from an aqueous solution; (2) standard septum cap of the GC with its central hole enlarged to $2.4 \mathrm{~mm}$; (3) stainless-steel insert in the phenolic cap of a standard reagent bottle; (4) epoxy glue applied to the outside surface of the cap around the insert; (5) Teflon gasket.

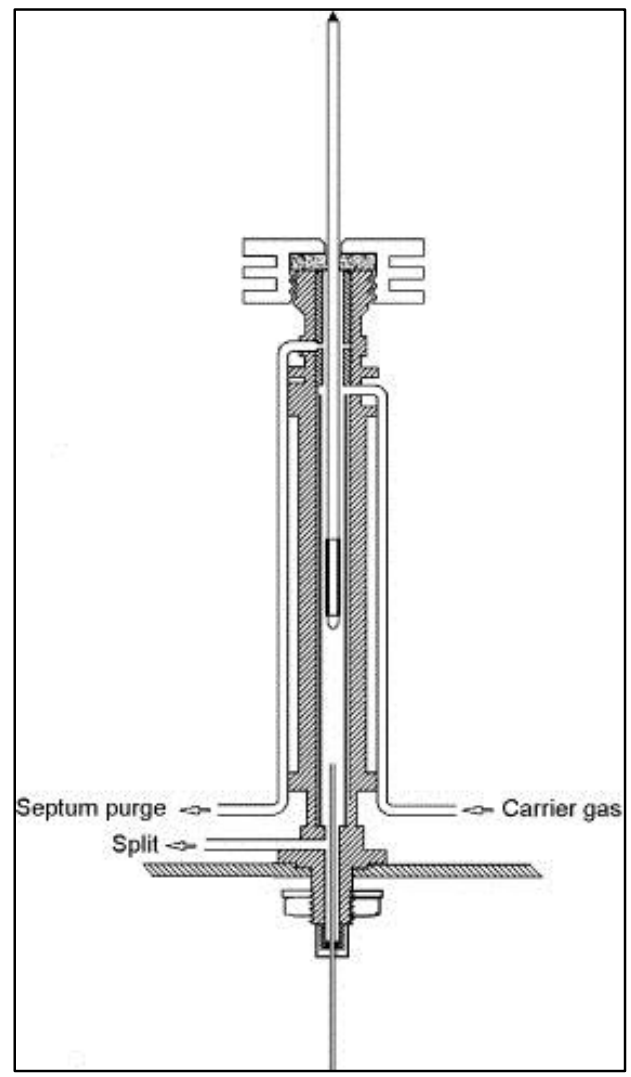

Fig. 2. SEP installed in a standard split/splitless injector. 


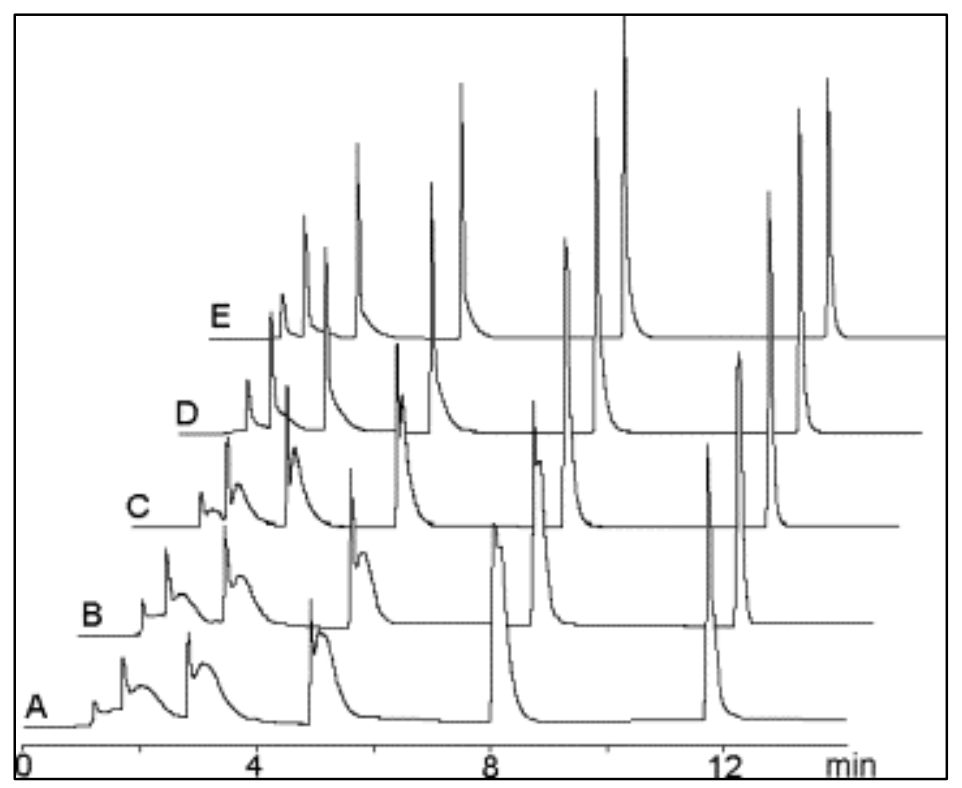

Fig. 3. Gas chromatograms of the homologous series of $n$-alkanes from $C_{5}$ to $C_{10}$ obtained with SEP sample introduction and with the injector operated under different conditions. Column A, $40 \mathrm{~m} \times 0.3 \mathrm{~mm} \times 0.25 \mu \mathrm{m}$ PS-089, programmed from 40 to $250{ }^{\circ} \mathrm{C}$ at $4{ }^{\circ} \mathrm{C} / \mathrm{min}$. Injector: (A) $220^{\circ} \mathrm{C}$, splitless, no flowless period; (B) $300{ }^{\circ} \mathrm{C}$, splitless, no flowless period; (C) $220^{\circ} \mathrm{C}$, split $15 \mathrm{ml} / \mathrm{min}$, no flowless period; (D) $220^{\circ} \mathrm{C}$, split $30 \mathrm{ml} / \mathrm{min}$, no flowless period; (E) $220^{\circ} \mathrm{C}$, split $15 \mathrm{ml} / \mathrm{min}$, flowless period $15 \mathrm{~s}$.

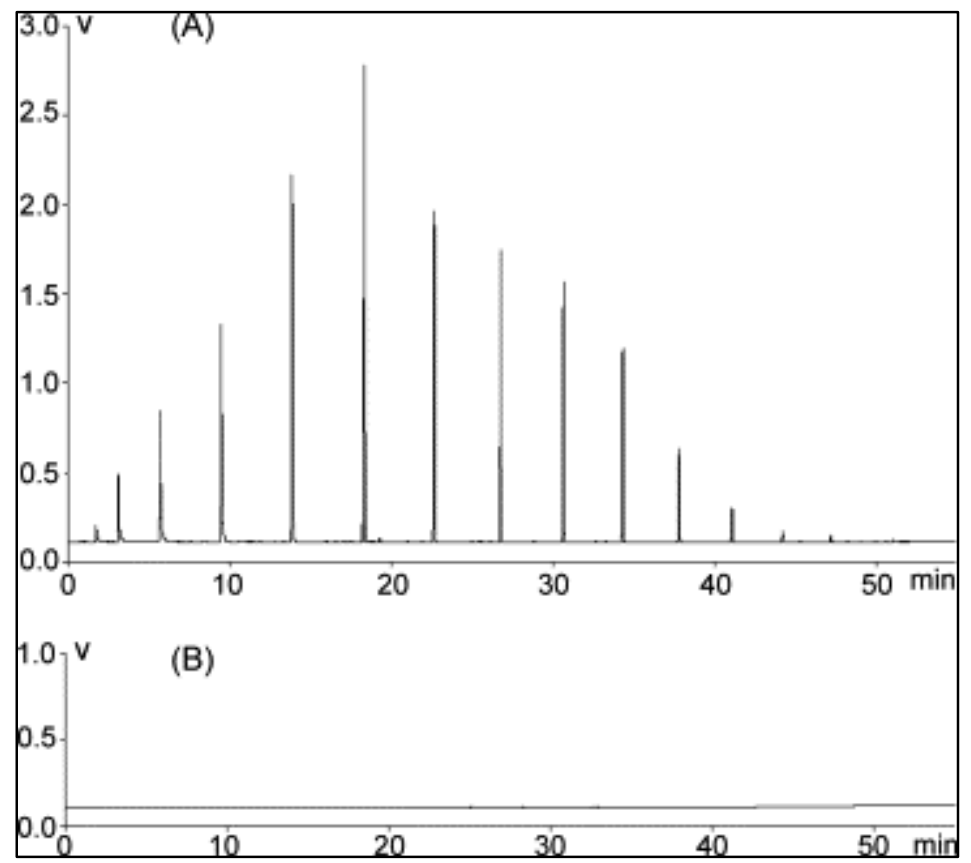

Fig. 4. (A) Gas chromatogram of $14 n$-alkanes from $C_{5}$ to $C_{18}$ enriched with a SEP for 10 min at $22^{\circ} \mathrm{C}$ from argon spiked with the alkanes at concentrations of about $1 \mu \mathrm{g} / \mathrm{I}$. Column B, $33 \mathrm{~m} \times 0.32 \mathrm{~mm}$ I.D. $\times 1.2 \mu \mathrm{m}$ PS-255, programmed from 30 to $250{ }^{\circ} \mathrm{C}$ at $4{ }^{\circ} \mathrm{C} / \mathrm{min}$, injector $230^{\circ} \mathrm{C}$, split $15 \mathrm{ml} / \mathrm{min}$, flowless period $15 \mathrm{~s}$; (B) blank analysis with the same, freshly conditioned SEP. The operational conditions are identical to those in (A). 


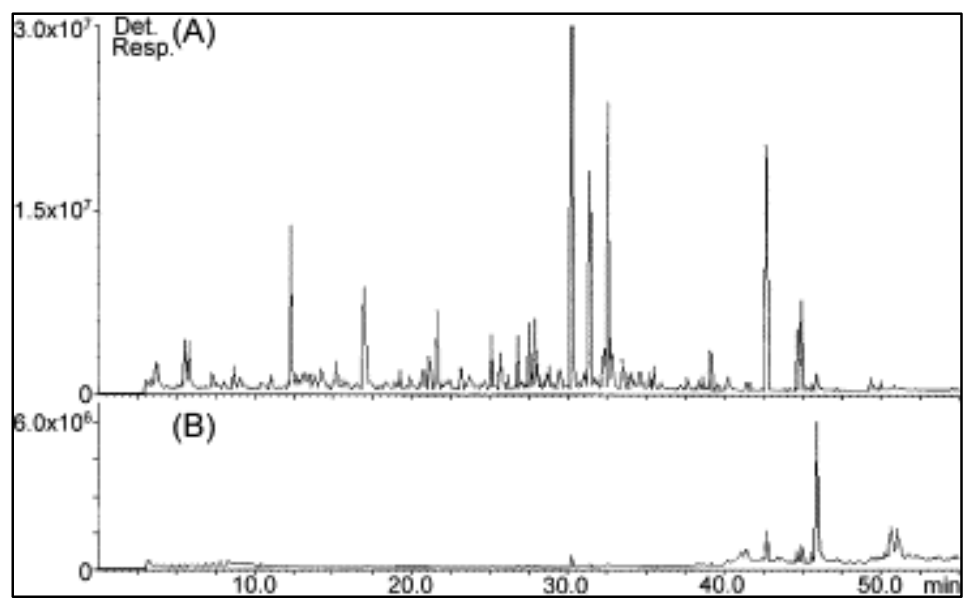

Fig. 5. Total ion chromatograms (TICS) of the headspace volatiles of dry fermented Rooibos tea enriched for $24 \mathrm{~h}$ at $40{ }^{\circ} \mathrm{C}$. Column A, $40 \mathrm{~m} \times 0.3 \mathrm{~mm}$ I.D. $\times 0.25 \mu \mathrm{m}$ PS-089, programmed from 30 to $280^{\circ} \mathrm{C}$ at $4{ }^{\circ} \mathrm{C} / \mathrm{min}$, injector $220^{\circ} \mathrm{C} ;$ split $15 \mathrm{ml} / \mathrm{min}$. (A) SEP-GC-MS, flowless period $15 \mathrm{~s}$; (B) SPME-GC-MS, $100 \mu \mathrm{m}$ PDMS.

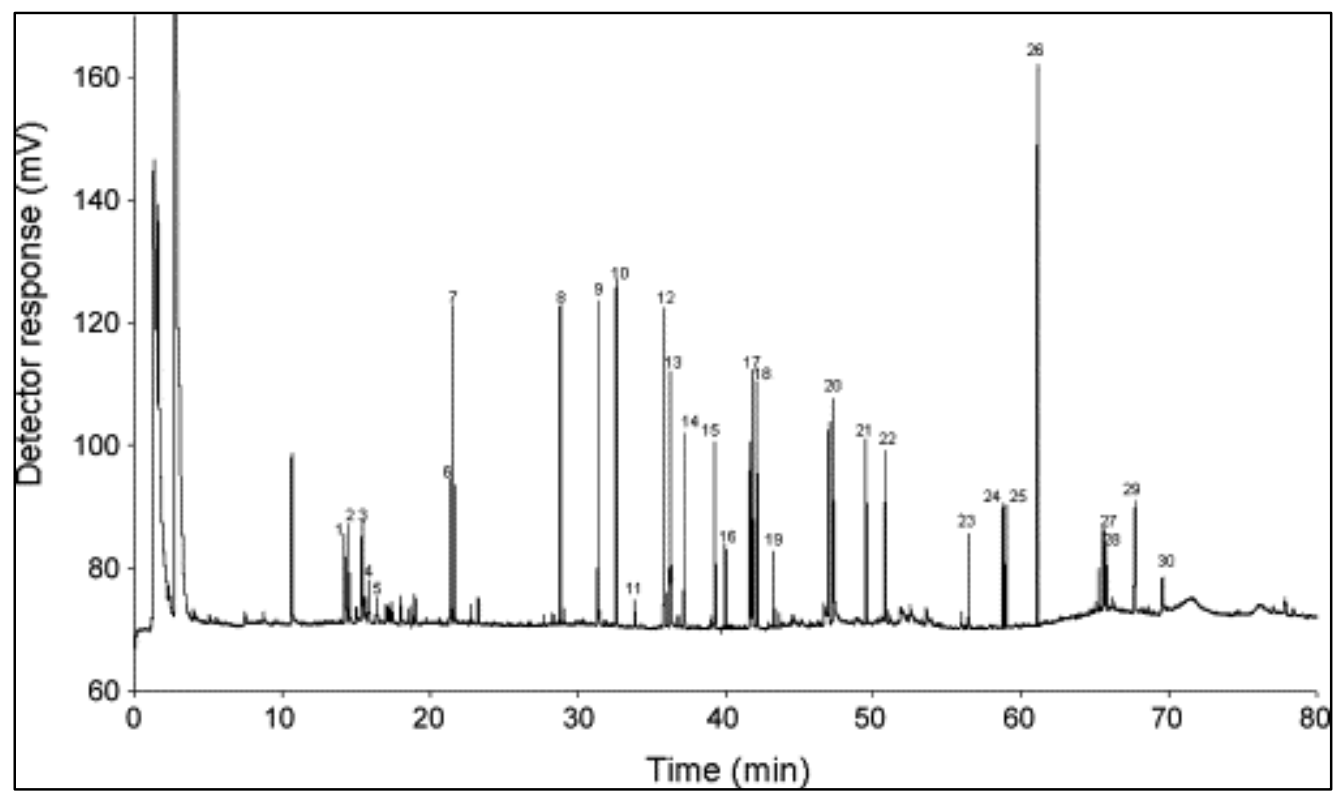

Fig. 6. Gas chromatogram of an aqueous sample containing the contaminants listed in Table 3 at concentrations of about $1 \mathrm{ng} / \mathrm{l}$. Sample enrichment for $1 \mathrm{~h}$ at $30^{\circ} \mathrm{C}$. Column A, $40 \mathrm{~m} \times 0.3 \mathrm{~mm} \mathrm{I.D.} \times 0.25 \mu \mathrm{m}$ PS-089, programmed at $5{ }^{\circ} \mathrm{C}$ from 35 to $280{ }^{\circ} \mathrm{C}$ (hold), injector $220^{\circ} \mathrm{C}$, split $15 \mathrm{ml} / \mathrm{min}$, flowless period $15 \mathrm{~s}$.

\section{References}

1. M. Biedermann, A. Fiscalini, K. Grob J. Sep. Sci., 27 (2004), p. 1157

2. K. Grob, A. Habich J. Chromatogr., 321 (1985), p. 45

3. B.V. Burger, M. le Roux, W.J.G. Burger J. High Resolut. Chromatogr., 13 (1990), p. 777

4. C.L. Arthur, J. Pawliszyn Anal. Chem., 62 (1990), p. 2145

5. E. Baltussen, P. Sandra, F. David, C. Cramers J. Microcolumn Sep., 11 (1999), p. 737

6. J. Pettersson, A. Kloskowski, C. Zaniol, J. Roeraade J. Chromatogr. A, 1033 (2004), p. 339

7. M. Ishikawa, O. Ito, S. Ishizaki, Y. Kurobayashi, A. Fujita Flavour Frag. J., 19 (2004), p. 183

8. B. Kolahgar, A. Hoffmann, A.C. Heiden J. Chromatogr. A, 963 (2002), p. 225 


\section{SAMPLE ENRICHMENT PROBE}

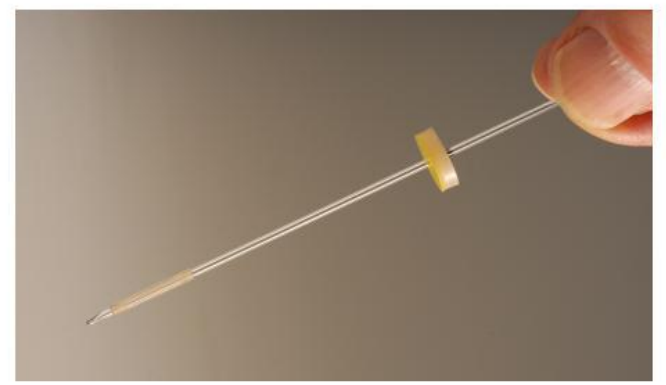

$15 \mathrm{~mm} \times 1.0 \mathrm{~mm} \mathrm{ID} \times 1.5 \mathrm{~mm}$ OD PDMS on a $1.2-\mathrm{mm}$ glass rod

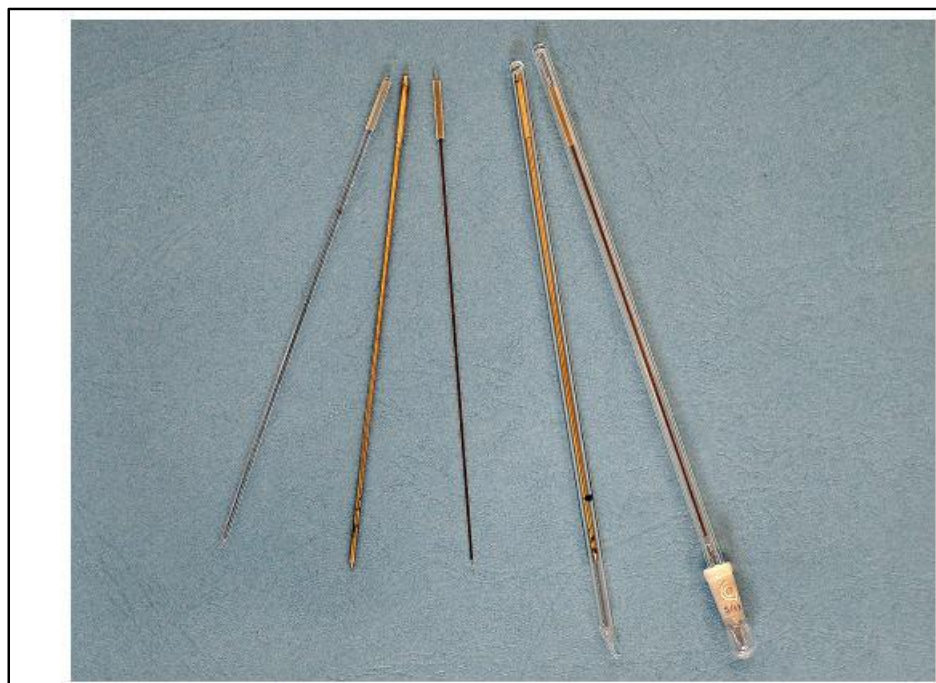

From left: SEPs with stainless steel, gold-plated stainless steel, fused silica shafts, and sealed off in glass tubes (on the far right with a B5 socket)

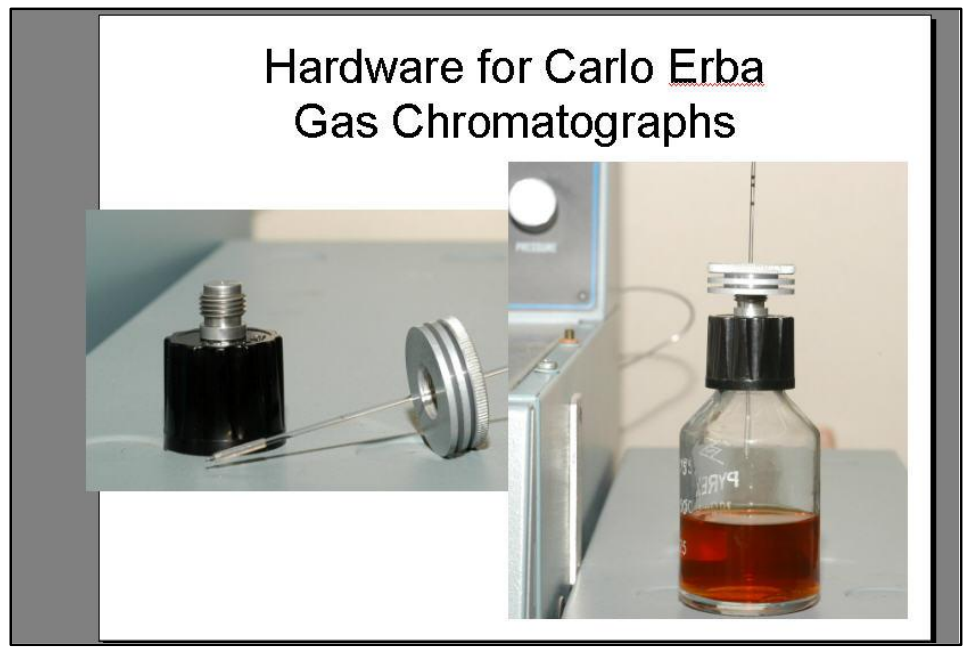




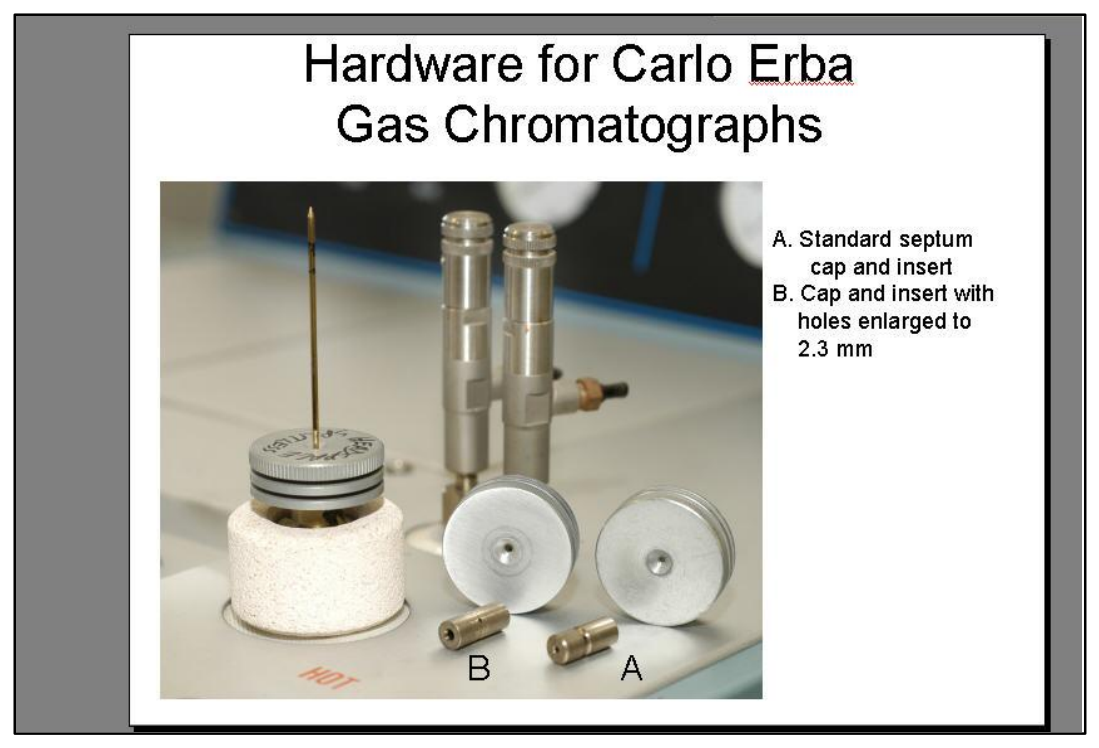

\section{Different Sampling Methods}

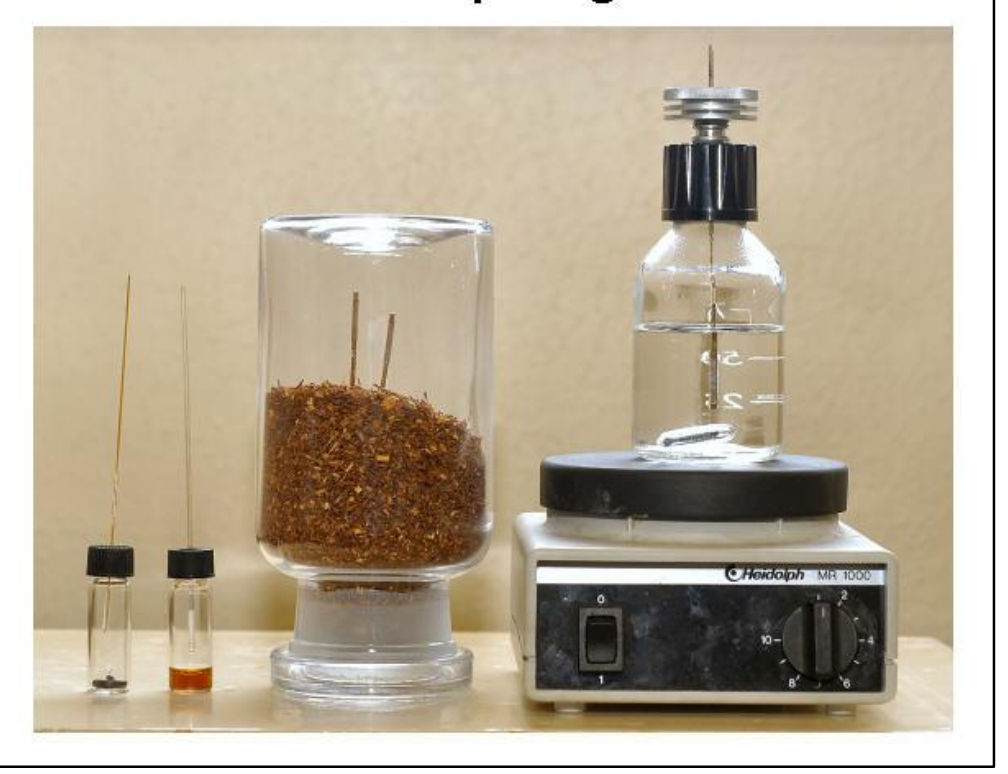

